\title{
Rhinosinusitis and nasal polyposis in aspirin sensitive and aspirin tolerant patients: are they different?
}

\author{
Marek L Kowalski
}

Hypersensitivity to aspirin (acetylsalicylic acid; ASA) is present in $5-10 \%$ of asthmatic patients and is associated with chronic rhinosinusitis and nasal polyposis, a syndrome referred to as the aspirin triad. ${ }^{12}$ The mechanism of the hypersensitivity reaction to aspirin is not immunological, but may be related to the inhibition of cyclo-oxygenase, an enzyme responsible for prostaglandin synthesis, by aspirin. ${ }^{2} \mathrm{~A}$ positive history of aspirin hypersensitivity, which is the hallmark of this syndrome, is a significant and reliable indicator of chronic intractable rhinosinusitis, persistent asthma of greater than average severity, and higher than ordinary medication requirements, including dependence on steroids. ${ }^{13}$ In addition to bronchospasm, most aspirin sensitive asthmatic subjects challenged with aspirin develop nasal symptoms including rhinorrhoea, sneezing and nasal obstruction, but the nasal responses to aspirin are superimposed on the existing pathology of the upper respiratory tract. The incidence of sinusitis identified by radiography in aspirin sensitive asthmatics may be up to $96.2 \%$ and the frequency of nasal polyps may be as high as $70.8 \%{ }^{24}$ compared with $6.7 \%$ in the general asthmatic population. ${ }^{5}$ A subgroup of aspirin sensitive patients has a reaction exclusively in the upper respiratory tract. They do not have asthma but the clinical picture of nasal disease (hypertrophic rhinosinusitis) in these patients is similar to that observed in patients with the aspirin triad. ${ }^{6}$

This article reviews the similarities and differences in the severity, pathophysiology, and management of upper airway disease in aspirin sensitive and aspirin tolerant patients with asthma and chronic rhinosinusitis.

\section{Is the rhinosinusitis more severe in aspirin sensitive patients?}

It is generally assumed that chronic rhinosinusitis in aspirin sensitive asthmatic patients is more severe than in aspirin tolerant patients. A high recurrence of nasal polyps and frequent need for sinal endoscopic surgery is characteristic of this group of patients. ${ }^{47}$ However, no quantitative study has been published comparing the extent and severity of the mucosal inflammation in aspirin sensitive patients with that in aspirin tolerant patients. Current technology offers new diagnostic methods for assessing the paranasal sinus disease, including computed tomographic (CT) scanning. CT scanning is the best means of visualising the anatomy and pathology of the sinuses, and allows assessment of the extent of sinus involvement and measurement of mucosal thickening in all sinuses including the ostiomeatal complex. We have recently introduced and validated a semiquantitative scoring system to assess the extent of sinus mucosal involvement, ${ }^{8}$ which is a modification of the scoring scale described by Newman et al. ${ }^{9}$ Using this CT scoring system, we have studied a group of 13 aspirin sensitive patients with chronic rhinosinusitis and nasal polyps and 11 patients with chronic rhinosinusitis and nasal polyps, but without intolerance to aspirin (Kowalski et al, in preparation). Both groups of patients had rhinosinusitis of similar duration and had undergone a similar number of nasal polypectomies. CT analysis showed signs of mucosal disease including mucosal swelling, hypertrophy, polyp formation in the sinuses, and obstruction of the ostiomeatal complex in both groups of patients. However, the extent of the disease, assessed by the scoring scale, was significantly higher in aspirin sensitive patients than in those with no sensitivity to aspirin. The differences were also significant for individual sinuses. This study has therefore shown, for the first time in a quantitative manner, that the extent of hyperplastic rhinosinusitis was greater in aspirin sensitive than in aspirin tolerant asthmatic subjects with rhinosinusitis of similar clinical severity.

Are the mechanisms of mucosal inflammation in the upper airways of aspirin sensitive and aspirin tolerant patients different?

It is not clear if the basic mechanism of chronic airway inflammation in aspirin sensitive patients is different from that in aspirin tolerant patients with chronic rhinosinusitis and nasal polyps. Nasal eosinophilia is a prominent feature of aspirin sensitive rhinosinusitis although quantitative differences from allergic rhinitis could not be consistently demonstrated. ${ }^{10}$ Although nasal polyps in aspirin sensitive patients are typically eosinophilic, their histological appearance does not seem to differ from polyps removed from aspirin tolerant patients. ${ }^{11}$ Intranasal challenge with aspirin results in an influx of eosinophils into the nasal lavage fluid, with concomitant increases in the concentration of eosinophilic cationic protein (ECP) and tryptase indicating specific activation of eosinophils and mast cells, respectively, in aspirin sensitive patients only. ${ }^{12}$ On the other hand, analysis of nasal lavage fluid showed no differences between the mean concentrations of vascular protein (albumin), secretory proteins (lactoferrin and lysozyme), or other inflammatory mediators (histamine, $\mathrm{PGD}_{2}$,
Correspondence to: kowalsml@psk2.am.lodz.pl 
$\left.\mathrm{LTC}_{4} / \mathrm{LTD}_{4}\right)$ in basal secretions of both aspirin sensitive and aspirin tolerant patients. ${ }^{13}$ However, oral or nasal challenge with aspirin resulted in a significant increase in the $\mathrm{LTC}_{4} / \mathrm{LTD}_{4}$ concentration in nasal washes, indicating activation of the lipoxygenase pathway of arachidonic acid metabolism in the nasal mucosa. ${ }^{13}{ }^{14}$ In contrast to earlier immunopathological examinations which showed that nasal polyps and sinus tissue from asthma sensitive patients were indistinguishable from those from aspirin tolerant patients, ${ }^{1011} 15$ recent studies of biopsy specimens from nasal polyps have provided new data..$^{16}$ Although aspirin tolerant allergic and non-allergic patients (most with aspirin sensitivity) had similar numbers of inflammatory cells in the polyp tissue (with the exception of $\mathrm{CD} 3+\mathrm{T}$ lymphocytes), both groups had distinct cytokine profiles. Interleukin 4 expression was predominant in the polyps of allergic subjects and expression of interferon gamma (IFN $\gamma$ ) was characteristic of those from non-allergic subjects. Moreover, sensitivity to aspirin was strongly correlated with non-allergic polyps and with production of the non-allergic profile of cytokines including IFN $\gamma$. These data suggest that the basic mechanism of chronic airway inflammation may be different in aspirin sensitive and aspirin tolerant patients with hyperplastic rhinosinusitis.

The immunological mechanisms underlying the more severe rhinosinusitis and recurrence of nasal polyps in aspirin sensitive patients remain unclear. In addition to enhanced inflammatory cell recruitment resulting from possible enhanced generation of chemotactic factors in the mucosa, delayed programmed cell death (apoptosis) may account for the increased accumulation of inflammatory cells in nasal polyp tissue. Because of their abundance in nasal polyps, eosinophils seem to be the primary inflammatory cells regulating and perpetuating inflammation. ${ }^{18}$ It has been suggested that eosinophils cultured from nasal polyps have increased survival because of delayed apoptosis compared with cells from control mucosa. In order to elucidate the possible involvement of delayed apoptosis in the pathogenesis of nasal polyposis in aspirin sensitive patients we used the TUNEL technique to detect apoptotic cells in paraffin sections of nasal polyps from 16 aspirin sensitive patients and 36 patients with no aspirin sensitivity. ${ }^{19}$ Using a computerised image analysis system we found that the number of eosinophils was significantly increased in nasal polyps from aspirin sensitive patients compared with aspirin tolerant patients. On the other hand, the number of apoptotic cells was significantly lower in aspirin sensitive patients (mean 5.5 (SE 1.5) cells $/ \mathrm{mm}^{2}$ ) than in either atopic (18.7 (3.8) cells $\left./ \mathrm{mm}^{2}\right)$ or non-atopic (21.3 (5.2) cells $/ \mathrm{mm}^{2}$ ) patients with nasal polyps. We therefore concluded that enhanced nasal eosinophilia, as well as severity and persistence of nasal polyposis, might be related to impaired apoptosis in aspirin sensitive patients. Eosinophil apoptosis is controlled by several cytokines and it is conceivable that the abovementioned cytokine imbalance may be involved in the pathogenesis of the persistent eosinophilic inflammation observed in the airway mucosa of aspirin sensitive patients.

We have recently reported that nasal polyp epithelial cells cultured from aspirin sensitive patients generated significantly less prostaglandin $\mathrm{E}_{2}\left(\mathrm{PGE}_{2}\right)$, both spontaneously and after stimulation with calcium ionophore, than cells cultured from polyps from aspirin tolerant patients. ${ }^{20}$ Our observation is further supported by the study of Picado et $a l^{21}$ who found lower expression of cyclo-oxygenase 2 in nasal polyp tissue from aspirin sensitive patients than in polyp tissue from aspirin tolerant patients. Because $\mathrm{PGE}_{2}$ has significant antiinflammatory activity, including an inhibitory effect on eosinophil chemotaxis and activation, we speculated that an intrinsic defect in local generation of $\mathrm{PGE}_{2}$ may contribute to the more severe eosinophilic inflammation in aspirin sensitive patients.

Is the management of chronic rhinosinusitis and nasal polyps different in aspirin sensitive patients?

Although aspirin is only one of many triggers exacerbating symptoms of rhinosinusitis and asthma in these patients, the presence of aspirin sensitivity heralds severe and protracted disease of the respiratory tract, requiring comprehensive management of all components of the syndrome.

The management of aspirin sensitive rhinosinusitis does not differ from treatment of other forms of this disease but there are several important points which have to be taken into account. Patient education and careful avoidance of aspirin and non-steroidal antiinflammatory drugs (NSAIDs) is very important since aspirin may cause severe asthmatic attacks in a significant proportion of patients admitted to the intensive care unit. Careful management of chronic rhinosinusitis is essential and may be a prerequisite for the improvement in bronchial symptoms. ${ }^{22}$ Traditional medicines such as nasal decongestants and antihistamines give limited relief in these patients, but topical steroids seem to be very effective. In many patients various surgical procedures may be needed to relieve chronic sinusitis and to remove nasal polyps. CT imaging for assessment of the extent of the sinus disease may also be useful. Depending on the stage of the disease, surgery consists of polypectomy, functional endoscopic sinus surgery, or ethmoidectomy in resistant cases. However, because surgery does not affect the underlying inflammatory component of the disease, medical treatment with topical steroids is also necessary following surgery.

A new approach to the treatment of asthma sensitive rhinosinusitis/asthma is to give aspirin orally after desensitisation since these patients can be desensitised by repeated challenges with aspirin and can then take the drug with impunity. ${ }^{23}$ In a subgroup of patients ingestion of aspirin after desensitisation may result in alleviation of nasal symptoms and decreased recurrence of nasal polyps. ${ }^{24}{ }^{25}$ Desensitisation 
can be also achieved after repeated intranasal application of lysine aspirin. It has been reported that intranasal desensitisation and prolonged treatment with soluble intranasal aspirin has a beneficial effect on the symptoms of chronic rhinosinusitis. Moreover, Patriarca et $a l^{26}$ reported that the weekly use of intranasal aspirin after desensitisation in patients with aspirin sensitivity significantly reduced the recurrence rate of nasal polyps compared with placebo. Interestingly, similar results were obtained after treatment with intranasal aspirin in aspirin tolerant patients with nasal polyps.

Leukotriene receptor antagonists and synthesis inhibitors have been shown to be of clinical benefit in patients with aspirin sensitive asthma. ${ }^{27} \mathrm{~A}$ preliminary study suggests that anti-leukotriene drugs may also be effective in the management of chronic rhinosinusitis in aspirin sensitive patients. ${ }^{28}$

In conclusion, available data suggest that chronic rhinosinusitis and nasal polyposis in patients with aspirin sensitivity differ with respect to both severity and basic pathophysiology from upper airway disease in aspirin tolerant patients. These differences should be taken into account when planning the management of rhinosinusitis in aspirin sensitive patients.

1 Samter M.Beers RF. Intolerance to aspirin: clinical studies and consideration of its pathogenesis. Ann Intern Med and consideration

2 Szczeklik A, Stevenson DD. Aspirin-induced asthma: advances in pathogenesis and management. F Allergy Clin Immunol 1999;104:5-13.

3 Kowalski ML. Management of aspirin-sensitive rhinosinusitis asthma syndrome: what role for aspirin desensitization? Allergy Proc 1992;13:175-84

4 Jantti-Alanko S, Holopainen E, Malmberg H. Recurrence of nasal polyps after surgical treatment. Rhinology 1989;8:5964.

5 Settipane GA. Nasal polyps: epidemiology, pathology, immunology and treatment. Am f Rhinol 1987;1:119-26.

6 Lumry WR, Curd JG, Zeiger RS. Aspirin-sensitive rhinosinusitis: the clinical syndrome and effects of aspirin administration. F Allergy Clin Immunol 1983;71:580-7.

7 McFadden EA, Kany RJ, Fink JN, et al. Surgery for sinusitis and aspirin triad. Laryngoscope 1990;100:1043-6.

8 Kordek P, Kowalski ML, Studniarek M, et al. CT staging of chronic hyperplastic rhinosinusitis. Przeglad Lek 2000;57: 150-3.

9 Newman LJ, Platts-Mills TAE, Phillips CD, et al. Chronic sinusitis: relationship of computed tomographic findings to allergy, asthma and eosinophilia. $\mathcal{F A M A}$ 1994;271:363-7.
10 Halopainen E, Makinen J, Paavolainen M. Nasal polyposis: relationship to allergy and acetylsalicylic acid intolerance. Acta Otolaryngol 1979;87:330-4.

11 Jankowski R, Bene MC, Moneret-Vautrin AD. Immunohistorical characteristics of nasal polyps. A comparison with healthy mucosa and chronic sinusitis. Rhinology 1989; 8(Suppl):51-8.

12 Kowalski ML, Grzegorczyk J, Wojciechowska B, et al. Intranasal challenge with aspirin induces cell influx and activation of eosinophils and mast cells in nasal secretions of ASA-sensitive patients. Clin Exp Allergy 1996;26:1-8.

13 Kowalski ML, Sliwinska-Kowalska M, Igarashi Y, et al. Nasal secretion in response to acetylsalicylic acid. f Allergy Clin Immunol 1993;91:580-98.

14 Picado C, Ramis I, Rosello J, et al. Release of peptide leukotriene into nasal secretions after local instillation of aspirin in sensitive asthmatic patients. Am Rev Respir Dis 1992;145:65-59.

15 Kowalski ML. Aspirin-sensitive rhinosinusitis/asthma syndrome: pathophysiology and management. Allergy Clin Immunol 1996;8:49-56.

16 Hamilos DL, Leung DY, Wood R, et al. Evidence for distinct cytokine expression in allergic versus nonallergic chronic cytokine expression in allergic versus nonallergic

17 Park HS, Nahm DH, Park K, et al. Immunohistochemical characterization of cellular infiltrate in nasal polyp from aspirin-sensitive asthmatic patients. Ann Allergy Asthma Immunol 1998;81:219-24.

18 Bachert C, Gevaert P, van Cauwenberge P. Nasal polyposis: a new concept on the formation of polyps. ACI International 1999;11:130-5.

19 Kowalski ML, Grzegorczyk J, Pawliczak R, et al. Decreased apoptosis in nasal polyps from patients with ASA sensitive rhinosinusitis/asthma (ASRSA), F Allergy Clin Immunol 1999;103:247.

20 Kowalski ML, Pawliczak R, Wozniak J, et al. Differential metabolism of arachidonic acid in nasal polyp epithelial metabolism of arachidonic acid in nasal polyp epithelial patients. Am f Respir Crit Care Med 2000;161:391-8.

21 Picado C, Fernandez-Morata JC, Juan M, et al. Cyclooxygenase-2 mRNA is downexpressed in nasal polyps from aspirin-sensitive asthmatics. Am f Respir Crit Care Med 1999;160:291-6.

22 Senior BA, Kennedy DW. Management of sinusitis in the asthmatic patient. Ann Allergy Asthma Immunol 1996;77:619.

23 Vane JR. Inhibition of prostaglandin synthesis as a mechanism of action for aspirin-like drugs. Nature New Biol 1971;231:232-5.

24 Sweet JA, Stevenson DD, Simon RA, et al. Long term effects of aspirin desensitization treatment for aspirin sensitive rhinosinusitis asthma. $\mathcal{F}$ Allergy Clin Immunol 1990;86:59-65.

25 Stevenson DD, Hankammer MA, Mathison DA. Long term ASA desensitization-treatment of aspirin sensitive asthmatic patients: clinical outcome studies. 7 Allergy Clin Immunol 1996;98:751-8.

26 Patriarca G, Bollioni P, Nucera E, et al. Intranasal treatment with lysine actylsalicylate in patients with nasal polyposis. Ann Allergy 1991;67:588-91.

27 Kuna P, Malmstrom K, Dahlen SE, et al. Montelukast (MK-0476), a CysLT receptor antagonist, improves asthma control in aspirin-sensitive asthmatic patients. $A m \mathcal{F}$ Respir Crit Care Med 1997;155:A975.

28 Dahlen SE, Nizankowska E, Dahlen B. The Swedish-Polish treatment study with the 5-lipoxygenase inhibitor Zileuton in aspirin-intolerant asthmatics. Am f Respir Crit Care Med 995;151:376-70. 